\title{
Experimental Human Pneumococcal Colonization in Older Adults Is Feasible and Safe, Not Immunogenic
}

\author{
Hugh Adler ${ }^{1,2}$, Esther L. German ${ }^{1}$, Elena Mitsi ${ }^{1}$, Elissavet Nikolaou ${ }^{1}$, Sherin Pojar ${ }^{1}$, Caz Hales ${ }^{1,2}$, Rachel Robinson ${ }^{1,2}$, \\ Victoria Connor ${ }^{1,2}$, Helen Hill ${ }^{1,2}$, Angela D. Hyder-Wright ${ }^{1,2,3}$, Lepa Lazarova ${ }^{1,2}$, Catherine Lowe ${ }^{1,2}$, Emma L. Smith ${ }^{1,2}$, \\ India Wheeler ${ }^{1,2}$, Seher R. Zaidi ${ }^{1,2}$, Simon P. Jochems ${ }^{1}$, Dessi Loukov ${ }^{1}$, Jesús Reiné ${ }^{1}$, Carla Solórzano-Gonzalez ${ }^{1}$, \\ Polly de Gorguette d'Argoeuves ${ }^{4}$, Tessa Jones ${ }^{4}$, David Goldblatt ${ }^{4}$, Tao Chen ${ }^{1}$, Stephen J. Aston ${ }^{2,5}$, Neil French ${ }^{2,5}$, \\ Andrea M. Collins ${ }^{1,2,6}$, Stephen B. Gordon ${ }^{1,7}$, Daniela M. Ferreira ${ }^{1 *}$, and Jamie Rylance ${ }^{1,2,7 *}$ \\ ${ }^{1}$ Department of Clinical Sciences, Liverpool School of Tropical Medicine, Liverpool, United Kingdom; ${ }^{2}$ Royal Liverpool and Broadgreen \\ University Hospitals NHS Trust, Liverpool, United Kingdom; ${ }^{3}$ Clinical Research Network, North West Coast, United Kingdom; ${ }^{4}$ University \\ College London Great Ormond Street Institute of Child Health, London, United Kingdom; ${ }^{5}$ Institute of Infection and Global Health, \\ University of Liverpool, Liverpool, United Kingdom; ${ }^{6}$ Aintree University Hospital, Liverpool, United Kingdom; and ${ }^{7}$ Malawi-Liverpool- \\ Wellcome Programme, Blantyre, Malawi
}

ORCID IDs: 0000-0003-4437-2298 (H.A.); 0000-0001-7586-6050 (E.M.); 0000-0003-4797-4710 (E.N.); 0000-0002-7746-3279 (S.P.);

0000-0002-3197-1657 (C.H.); 0000-0002-7994-501X (L.L.); 0000-0002-4835-1032 (S.P.J.); 0000-0002-3413-6884 (D.L.);

0000-0001-7462-2054 (J. Reiné); 0000-0001-9129-5569 (C.S.-G.); 0000-0002-0769-5242 (D.G.); 0000-0002-5489-6450 (T.C.);

0000-0003-4814-8293 (N.F.); 0000-0002-4094-1572 (A.M.C.); 0000-0001-6576-1116 (S.B.G.); 0000-0002-0594-0902 (D.M.F.);

0000-0002-2323-3611 (J. Rylance).

\section{Abstract}

Rationale: Pneumococcal colonization is key to the pathogenesis of invasive disease but is also immunogenic in young adults, protecting against recolonization. Colonization is rarely detected in older adults, despite high rates of pneumococcal disease.

Objectives: To establish experimental human pneumococcal colonization in healthy adults aged 50-84 years, to measure the immune response to pneumococcal challenge, and to assess the protective effect of prior colonization against autologous strain rechallenge.

Methods: Sixty-four participants were inoculated with Streptococcus pneumoniae (serotype 6B; 80,000 cfu in each nostril). Colonization was determined by bacterial culture of nasal wash, and humoral immune responses were assessed by anticapsular and antiprotein IgG concentrations.

Measurements and Main Results: Experimental colonization was established in $39 \%$ of participants $(25 / 64)$ with no adverse events. Colonization occurred in 47\% (9/19) of participants aged
$50-59$ compared with $21 \%(3 / 14)$ in those aged $\geqslant 70$ years. Previous pneumococcal polysaccharide vaccination did not protect against colonization. Colonization did not confer serotypespecific immune boosting, with a geometric mean titer $(95 \%$ confidence interval) of $2.7 \mu \mathrm{g} / \mathrm{ml}(1.9-3.8)$ before the challenge versus $3.0(1.9-4.7) 4$ weeks after colonization $(P=0.53)$.

Furthermore, pneumococcal challenge without colonization led to a drop in specific antibody concentrations from $2.8 \mu \mathrm{g} / \mathrm{ml}(2.0-3.9)$ to $2.2 \mu \mathrm{g} / \mathrm{ml}(1.6-3.0)$ after the challenge $(P=0.006)$. Antiprotein antibody concentrations increased after successful colonization. Rechallenge with the same strain after a median of 8.5 months (interquartile range, 6.7-10.1) led to recolonization in 5/16 $(31 \%)$.

Conclusions: In older adults, experimental pneumococcal colonization is feasible and safe but demonstrates different immunological outcomes compared with younger adults in previous studies.

Keywords: Streptococcus pneumoniae; human challenge models; immunity; elderly; vaccination

(Received in original form April 30, 2020; accepted in final form September 17, 2020)

*These authors are joint last authors.

Supported by the Bill and Melinda Gates Foundation (grant number OPP1117728) and U.K. Medical Research Council (grant number MR/M011569/1). The funders had no role in study design, data analysis, or the decision to submit for publication.

Author Contributions: Obtained funding: S.B.G. and D.M.F. Study design: H.A., S.B.G., D.M.F., and J. Rylance. Obtained ethical approval: H.A. and J. Rylance. Participant recruitment, clinical procedures, and data collection: H.A., C.H., R.R., V.C., H.H., A.D.H.-W., L.L., C.L., E.L.S., I.W., and S.R.Z. Data analyses: H.A., E.L.G., E.M., E.N., S.P., S.P.J., D.L., J. Reiné, C.S.-G., P.d.G.d'A., T.J., D.G., T.C., and D.M.F. Study oversight: S.J.A., N.F., A.M.C., S.B.G., D.M.F., and J. Rylance. All authors contributed to the manuscript and approved the final version submitted for publication.

Correspondence and requests for reprints should be addressed to Daniela M. Ferreira, Ph.D., Respiratory Infection Group, Liverpool School of Tropical Medicine, Liverpool L3 5QA, UK. E-mail: daniela.ferreira@Istmed.ac.uk.

This article has a related editorial.

This article has an online supplement, which is accessible from this issue's table of contents at www.atsjournals.org.

Am J Respir Crit Care Med Vol 203, Iss 5, pp 604-613, Mar 1, 2021

Copyright $\odot 2021$ by the American Thoracic Society

Originally Published in Press as DOI: 10.1164/rccm.202004-14830C on September 17, 2020

Internet address: www.atsjournals.org 


\section{At a Glance Commentary}

\section{Scientific Knowledge on the}

Subject: Colonization is rarely detected in older adults despite high rates of pneumococcal disease. We hypothesize that the susceptibility of elderly participants to pneumococcal infection could be associated with their lack of immune boosting to pneumococcal carriage.

\section{What This Study Adds to the Field:}

We have shown that pneumococcal carriage is possible in older adults and that carriage rates are not different from those of young adults. However, in contrast to what we have previously reported in young adults, carriage is not an immunizing event in older adults.

Older adults have high rates of morbidity and mortality from pneumococcal disease, including pneumonia and meningitis (1-4). Nasopharyngeal pneumococcal colonization necessarily precedes pneumococcal disease (5). Paradoxically, colonization has generally been considered to be infrequent in older adults, with rates ranging from $1.9 \%$ to $4.2 \%$ in studies employing culture-based methodology (6-8). This may be due to insensitive detection methods or an altered niche of colonization in older adults $(9,10)$. Others have suggested that older adults are inherently less susceptible to colonization, perhaps because of age-related dysregulation of airway inflammatory pathways (11-13). Colonization is immunogenic in young adults, protecting against future recolonization with the same serotype $(14,15)$. Therefore, reduced colonization or altered postcolonization immunity in older adults may partially explain their susceptibility to pneumococcal disease. The immune dynamics of colonization in older adults have not been previously studied.

In adults over 65 years of age, recommendations for pneumococcal polysaccharide vaccine (PPV23) and 13 -valent pneumococcal conjugate vaccine (PCV13) administration vary between countries $(16,17)$. Both vaccines induce anticapsular polysaccharide antibody production, although the functionality of antibodies produced is reduced in older adults $(18,19)$. Both vaccines confer a degree of protection against vaccineserotype invasive pneumococcal disease $(20,21)$, although a combination of imperfect efficacy and serotype replacement by nonvaccine serotypes have reduced the effectiveness of both vaccines (22). PCV13 confers short-term protection against colonization (23), most likely via antibody-mediated bacterial agglutination (24), whereas high-quality prospective data regarding PPV23 and colonization are lacking (25). Protection against colonization is a surrogate for protection against disease. Because neither vaccine confers complete protection against pneumococcal disease in older adults, improved vaccines and immunization strategies are urgently required.

Controlled human infection models have revolutionized the study of infectious disease pathogenesis (26). Such studies typically recruit healthy, young adults who are at low risk of severe disease or complications. Rhinovirus challenge studies have been performed in older participants, including those with respiratory comorbidities (27), but bacterial challenge studies have not been systematically attempted in older people. There is an unmet need for such research in this population given the higher age-specific incidence of pathogens such as pneumococcus and the uncertain generalizability from studies of young adults.

We report the expansion of the experimental human pneumococcal colonization (EHPC) model into adults aged $\geqslant 50$ years, aiming to establish the feasibility, susceptibility, and safety of EHPC in older adults. We also define the immunogenicity of pneumococcal colonization in this population (measured by antibody responses) and the protection conferred by primary challenge against subsequent recolonization by the same strain.

Some of the results of these studies have been previously reported in the form of a preprint (https://doi.org/10.1101/ 2020.04.23.20077073). This study was approved by the National Health Service Research Ethics Committee (16/NW/0031) and its registered International Standard Randomised Controlled Trial Number is 10948363.

\section{Methods}

Healthy adults aged 50-84 years were approached using advertisements, research volunteer databases, and primary care patient lists. All participants provided written informed consent and underwent safety screening, including review of primary care records, physical examination, electrocardiography, spirometry, complete blood count, and biochemical profile, before participation (see online supplement for full inclusion/exclusion criteria and study methods). In brief, participants were excluded if they had regular close contact with children aged $<5$ years or immunosuppressed adults, uncontrolled medical comorbidity, recent steroid or antibiotic therapy, significant smoking history, or history of culture-proven pneumococcal disease. Vaccination history was recorded; in the United Kingdom, PPV23 is recommended to all 65-yearolds, but PCV13 is not routinely offered. The study was overseen by an independent data monitoring and safety committee.

Experimental pneumococcal challenge was performed as previously described (15, 28). Baseline nasal wash and serum samples were taken up to 7 days before inoculation. Inoculation entailed installation of an estimated 80,000 cfu per nostril of Streptococcus pneumoniae serotype 6B (strain BHN418 [29]; GenBank accession number ASHP00000000.1) using a micropipette with the participant in a reclining chair. Participants recorded and communicated their temperature to the research team by text message every day for the following week. Nasal washes were repeated on Days 2, 7, 9, 14, 22, and 29 after inoculation, with a second serum sample on Day 29. After completion, if participants' nasal wash remained culture positive at Day 22 or Day 29 , they were treated with oral amoxicillin for 3 days. Those who had been colonized at any time point were invited to return up to 1 year later for rechallenge using the same pneumococcal strain, dose, and procedure. Repeat nasal washes for the rechallenge study were taken at baseline and at 2, 7, and 14 days after inoculation. The timeline is summarized in Figure 1.

Nasal wash samples were processed within an hour of collection and were incubated overnight on gentamicin/blood agar at $37^{\circ} \mathrm{C}$ in $5 \%$ carbon dioxide (an 


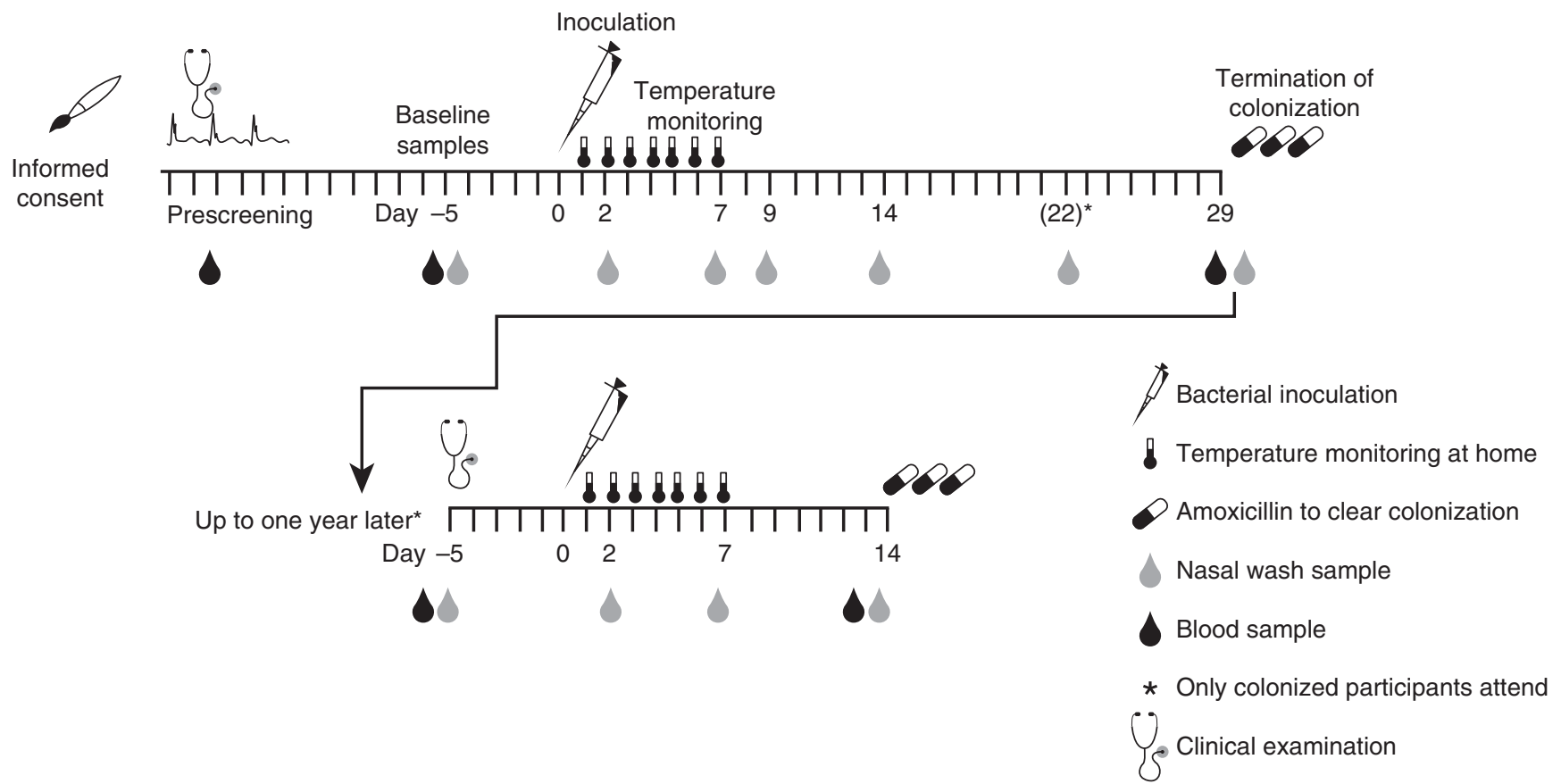

Figure 1. Timeline for the study, including the optional rechallenge (for participants who developed colonization during the primary study) up to 1 year later.

aliquot was also plated on nonselective blood agar to ensure accurate estimation of density). Experimental colonization was defined as the growth of serogroup 6 pneumococcus at any time point during the 29 days after inoculation and was identified using standard microbiological criteria (30). Anti-6B capsular polysaccharide (CPS) IgG concentrations in serum were measured using a modified World Health Organization ELISA protocol (see online supplement). Serum antibodies against 27 pneumococcal proteins were measured using multiplex electrochemiluminescence (Meso Scale Discovery [MSD]) as previously reported (15) (methodological details are reported in the online supplement).

\section{Statistical Analysis}

The primary endpoint was the rate of experimental colonization in older adults, which we compared with the rate in younger adults in other EHPC studies using the same methodology, performed during the same time period. We did not recruit a designated young control cohort for this study; more than 200 young adults took part in other EHPC studies during this time, with the same inoculation protocol.

The typical experimental colonization rate in young healthy adults is $45 \%$ (15), and we hypothesized on the basis of crosssectional colonization studies (6) that this would fall to $10 \%$ in older adults. A sample size of 64 would detect this difference in rates of experimental colonization at $\alpha=0.05$ with a power of 0.80 , allowing for a $10 \%$ dropout rate. We did not prespecify that we would exclude participants who were naturally colonized with pneumococcus at baseline but performed post hoc sensitivity analyses excluding such participants from colonization outcomes.

Secondary microbiological endpoints included colonization rates stratified by age, colonization rates in PPV23-vaccinated participants, colonization density and duration, and adverse events. Immunological endpoints included the change in antibody titer after challenge and the association of preexisting antibodies with the probability of colonization and colonization density. Total bacterial density during the study was defined as the area under the time-density curve (AUC), which calculated according to the trapezoid rule using values of $\left[\log _{10}(\right.$ bacterial density +1$\left.)\right]$ for each interval, with all participants assigned a density of $0 \mathrm{cfu} / \mathrm{mL}$ on inoculation day. For participants in the rechallenge phase of the study, colonization densities up to Day 14 after rechallenge were compared with the colonization densities over the same time period during the primary challenge.

Colonization rates in different groups were compared using $\chi^{2}$ or Fisher's exact test. Antibody results were log-transformed and compared between groups using the unpaired $t$ test or ANOVA or were compared within groups (before and after pneumococcal challenge) using the paired $t$ test, with results presented as geometric mean titers (GMTs) and 95\% confidence intervals (CIs). Correlations between continuous variables were assessed using Pearson's correlation. Nonparametric tests were used for comparisons of untransformed values within the (smaller) rechallenge cohort. The effects of baseline anti-6B antibodies, adjusted for age and sex, on the development of colonization or on colonization density AUC were assessed using logistic regression and linear regression, respectively. Fold changes in the 27 antiprotein antibodies between baseline and Day 29 were compared between colonized and noncolonized participants using multivariate regression. No imputations were made for missing data. All tests were two tailed, and a $P$ value $<0.05$ was considered significant. All analyses were performed using SPSS version 24 (IBM). 


\section{Results}

\section{Participant Characteristics}

The oldest participant was aged 80 years, and the median age was 64; the baseline characteristics of the cohort are given in Table 1 . The recruitment process and screening outcomes are outlined in Figure E1 in the online supplement. Ten participants were excluded on the basis of abnormal clinical findings at their prescreening visit, and no participants had previously received pneumococcal conjugate vaccine. Three were naturally colonized with $S$. pneumoniae at baseline, all of whom remained in the study and underwent experimental inoculation as per protocol.

\section{Experimental Colonization Rates}

The median inoculation dose was $84,333 \mathrm{cfu} / \mathrm{ml}$ (range, 69,333-92,833). Experimental colonization developed in 25 participants $(39.1 \%$; $95 \% \mathrm{CI}$, 28.1-51.3\%) (Figures 2 and 3 and Table E3). This was not significantly different from the $46.7 \%$ achieved in 225 young adults in the comparison group $(P=0.281)$ (Figure 3$)$. Colonization rates were $32 \%$ in men $(9 / 28)$ versus $44 \%$ $(16 / 36)$ in women $(P=0.32)$. Colonization rates within the over-50 cohort did not differ by age decile ( $\chi^{2}$ for trend,
$P=0.146)$, Figure 3 . When baseline natural carriers were excluded, the overall colonization rate was $37.7 \%$ (95\% CI, 26.6-50.3\%; $n=23 / 61$ )

Experimental colonization density and duration. Colonization densities varied substantially within and between participants; the average AUC of density was $34.4 \mathrm{cfu}$ days/ml (95\% CI, 19.9-48.9), and a graph of individual participants' colonization dynamics is in Figure E2. The median duration of colonization was 22 days, with $8 / 25$ participants still having detectable colonization at Day 29. Four participants had no detectable colonization after Day 2. AUC of density did not differ with age, either as assessed by age deciles (ANOVA, $P=0.84$ ) or by linear correlation with age $(r=-0.099 ; P=0.64)$.

\section{Experimental colonization in PPV23} recipients. Experimental colonization rates were not different in those who were previously immunized with PPV23 compared with those who were not $(8 / 22$ [36.4\%] vs. $17 / 42$ [40.5\%], respectively; $P=0.75)$. This pattern remained in sensitivity analyses restricted to participant over 65 years old and to the most recent vaccine recipients. We did not identify any significant predictors of colonization using logistic models, having tested PPV23 immunization status and other clinical and laboratory data (online supplement).

\section{Immunological Results}

Paired serum samples from baseline and 29 days were available for 62/64 participants. There were no differences in baseline antibody anti-CPS IgG titers between men and women (GMT, $2.82 \mu \mathrm{g} / \mathrm{ml} ; 95 \% \mathrm{CI}$, $1.91-4.18$ vs. GMT, $2.71 \mu \mathrm{g} / \mathrm{ml}$; 95\% CI, $1.99-3.69$, respectively; $P=0.87$ ). Previous PPV23 was associated with higher baseline antibody concentrations compared with no prior PPV23 (GMT, $4.17 \mu \mathrm{g} / \mathrm{ml}$; 95\% CI, 2.84-6.12 vs. GMT, $2.24 \mu \mathrm{g} / \mathrm{ml}$; 95\% CI, $1.67-2.99$, respectively; $P=0.01$ ). We did not find evidence of a fall in antibody concentrations over time after vaccination. In fact, there was a moderate positive correlation between PPV23 recipients' baseline antibody concentrations and time since vaccination $(r=0.46 ; P=0.034)$. Baseline antibody concentrations increased with age $(r=0.34 ; P=0.007)$, but this correlation was not evident when PPV23 recipients were excluded ( $r=0.19 ; P=0.24$; Figure E3).

\section{Anticapsular Antibodies and Experimental Colonization}

Higher baseline antibody concentrations were not associated with protection against experimental colonization. IgG GMTs in colonized and noncolonized participants were $2.71 \mu \mathrm{g} / \mathrm{ml}$ (95\% CI, 1.91-3.84) and $2.79 \mu \mathrm{g} / \mathrm{ml}$ (95\% CI, 2.0-3.9) respectively

Table 1. Baseline Characteristics of All Volunteers in the Study

\begin{tabular}{|c|c|c|c|c|}
\hline & \multicolumn{3}{|c|}{ Age-defined Risk Category } & \multirow[b]{2}{*}{ Total } \\
\hline & $50-64$ & $65-74$ & $75-84$ & \\
\hline Participants inoculated, $n$ & 34 & 25 & 5 & 64 \\
\hline Sex, F, $n(\%)$ & $18(52.9)$ & $15(60)$ & $3(60)$ & $36(56.3)$ \\
\hline $\begin{array}{l}\text { Age at inoculation, yr, median } \\
\text { (range) }\end{array}$ & $59(52-62)$ & $69(66-70)$ & $78(78-79)$ & $64(59-69)$ \\
\hline $\begin{array}{l}\text { Inoculation dose, cfu/ml, median } \\
\text { (range) }\end{array}$ & $83,833(76,000-90,167)$ & $84,333(69,333-92,833)$ & $84,750(73,833-89,167)$ & $84,333(69,333-92,833)$ \\
\hline Former smokers, $n(\%)$ & $10(29.4)$ & $11(44)$ & $2(40)$ & $22(34.4)$ \\
\hline $\begin{array}{l}\text { Smoking history, pack-years, } \\
\text { median (IQR) }\end{array}$ & $10(4.5-10)$ & $5(2-10)$ & $8(1-8)$ & $6.5(3.3-10)$ \\
\hline $\begin{array}{l}\text { Participants with any reported } \\
\text { comorbidity, } n(\%)^{*}\end{array}$ & $14(41.2)$ & $15(60)$ & $3(60)$ & $32(50.0)$ \\
\hline $\begin{array}{l}\text { Participants prescribed any regular } \\
\text { medication, } n(\%)\end{array}$ & $11(32.4)$ & $18(72)$ & $3(60)$ & $32(50.0)$ \\
\hline $\begin{array}{l}\text { Prior pneumococcal polysaccharide } \\
\text { vaccine, } n(\%)\end{array}$ & $0(0)$ & $17(68)$ & $5(100)$ & $22(34.4)$ \\
\hline $\begin{array}{l}\text { Naturally colonized at baseline, } n \\
\text { (serogroups) }\end{array}$ & $3(23,3,15)$ & 0 & 0 & 3 \\
\hline
\end{tabular}

Definition of abbreviation: IQR = interquartile range.

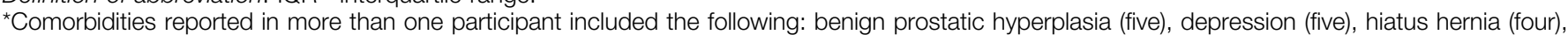

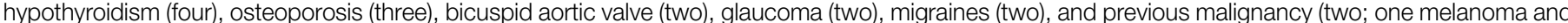
one testicular). 


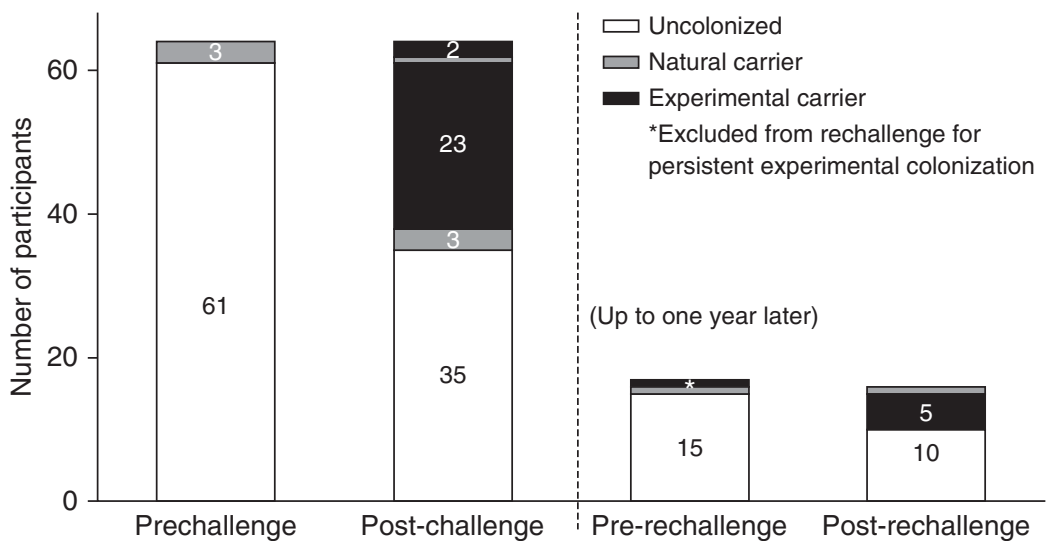

Figure 2. Microbiological status of participants before and after inoculation. Three participants were naturally colonized with non-6B serotypes at baseline, two of whom subsequently developed experimental colonization after inoculation (see online supplement for details); 23 previously uncolonized participants developed experimental colonization, and colonization with nonexperimental strains (serogroups 15, 20, and non-vaccine-type, Statens Serum Institut group G). At screening before rechallenge up to 1 year later, one volunteer was naturally colonized (with serogroup 15), and another was still colonized with the experimental strain; the latter was treated with amoxicillin and excluded from the remainder of the study. Five of the remaining 16 participants developed experimental colonization after rechallenge.

$(P=0.90)$. Adjustment for age and sex using logistic regression did not alter these findings (online supplement).

We did not observe an increase in antibody concentrations after colonization (Figure 4 and Table E5). Of interest, pneumococcal challenge without colonization led to diminished antibody concentrations; the GMT in subjects who did not develop experimental colonization fell from $2.79 \mu \mathrm{g} / \mathrm{ml}$ (95\% CI, 2.9-3.9) at baseline to $2.17 \mu \mathrm{g} / \mathrm{ml}(95 \% \mathrm{CI}, 1.57-3.0)$ at Day $29(P=0.006)$. On sensitivity analyses subdivided by PPV23 administration, this

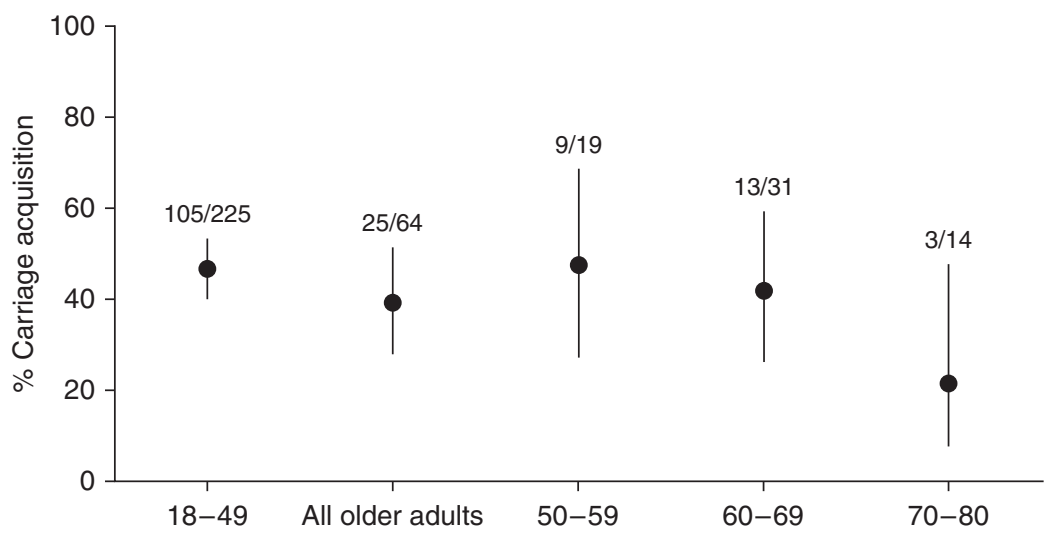

Figure 3. Experimental colonization rates in older adults (defined by positive pneumococcal culture in nasal wash at any time point after inoculation) compared with a young adult cohort (from similar studies conducted during the same time period) and broken down by age decile within the older cohort. Numbers denote (number colonized)/(total number in that age category). Error bars represent 95\% confidence intervals. There were no statistically significant differences between the older age category or subcategories and the younger volunteers. summarized as fold changes, there was no correlation with age in either colonized $(r=-0.025 ; P=0.906)$ or noncolonized participants $(r=-0.155 ; P=0.352)$. Higher baseline antibody concentrations did not correlate with lower colonization density, nor did higher density correlate with higher Day-29 antibody concentrations or greater fold change in antibody concentrations (online supplement).

\section{Antiprotein Antibodies}

Serum antibody concentrations against 27 pneumococcal proteins were measured at baseline and at Day 29 after the challenge using MSD multiplex electrochemiluminescence. There were no differences in the baseline concentrations of any antibody between colonized and noncolonized participants (Figure E4). In contrast to the anticapsular IgG response, antibody titers against several pneumococcal proteins were increased after pneumococcal colonization, including PspC, PspA-UAB055, RrgA-Tigr4, PiuA, and PcpA (all $P<0.001$ ) (Figure 5). In individuals who remained uncolonized, there was no significant change in any antiprotein antibodies.

\section{Mucosal Antibodies}

Anti-6B antibody concentrations in nasal wash were similar at baseline between colonized and noncolonized participants, and these concentrations did not change significantly after bacterial challenge (Figure E5).

\section{Protection against Experimental Recolonization}

Rechallenge was performed in 16 of the 25 participants who originally developed experimental colonization after a median interval of 259 days (interquartile range, 205-308 d; Figure E1). One was colonized with serogroup 15 pneumococcus at time of rechallenge and participated in the study as per protocol. A further participant was colonized with a serotype $6 \mathrm{~B}$ pneumococcus at time of rechallenge; he could not recall whether he had taken the 3-day course of amoxicillin prescribed after the initial study completion. Communityacquired colonization with serogroup 6 pneumococci is uncommon in Liverpool (31). The participant was not reinoculated but was treated with amoxicillin for 5 days. Subsequent nasal washes at 7 and 28 days were negative by both culture and PCR. 
A

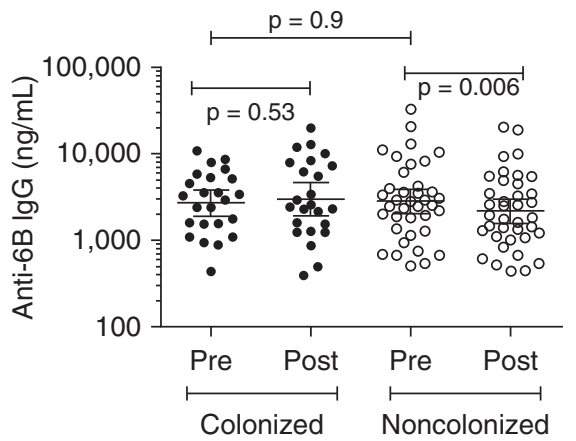

C

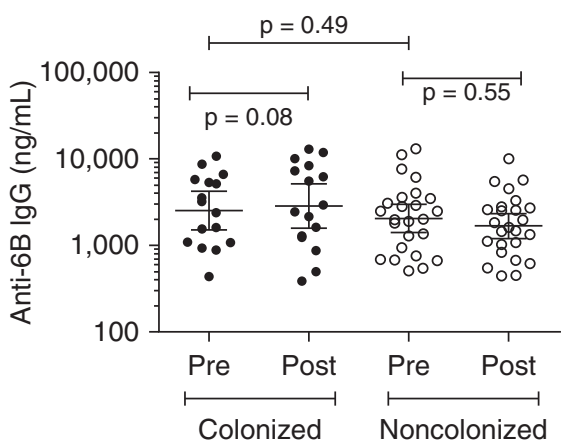

E

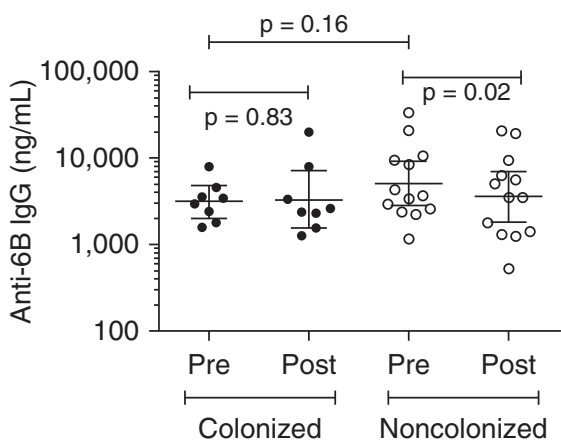

B

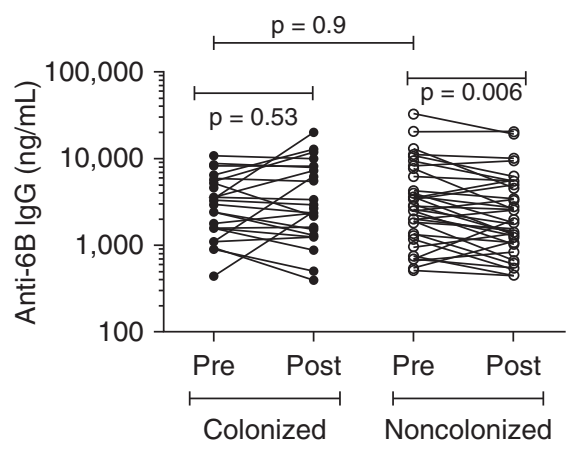

D

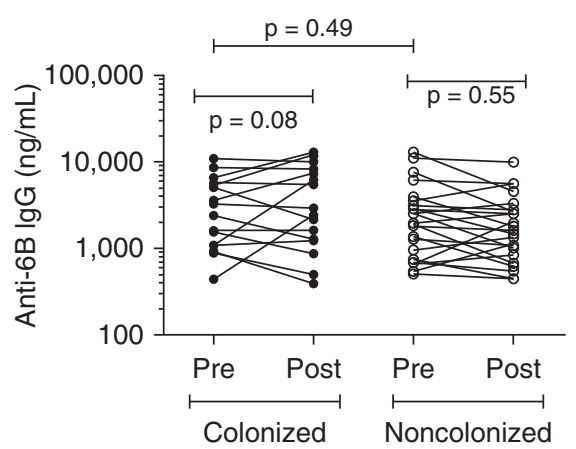

$\mathbf{F}$

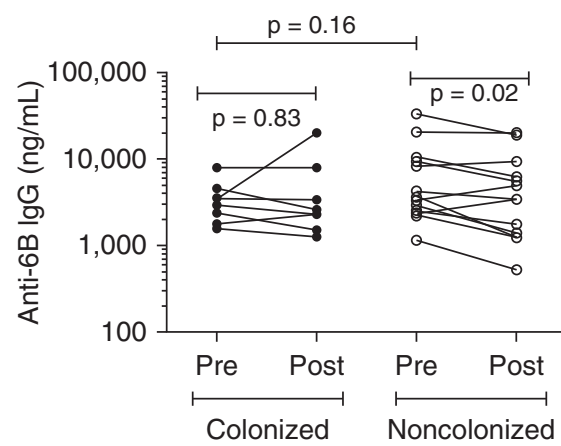

Figure 4. $(A-F)$ Anti-6B capsular polysaccharide lgG concentrations at baseline and Day 29 after inoculation in the full cohort $(A$ and $B)$, participants who had never received pneumococcal polysaccharide vaccine $(C$ and $D)$ and participants who had received pneumococcal polysaccharide vaccine ( $E$ and $F$ ). Each symbol represents a single participant. The lines and error bars in $A, C$, and $E$ represent geometric means (95\% confidence intervals); the lines in $B, D$, and $F$ connect the baseline and Day 29 values for each participant.

The median age of rechallenge participants was 63.5 years, and 11 (68.7\%) were female. Within 14 days of inoculation, colonization was detected in five participants (31.3\%).

\section{Colonization Density in Rechallenge Participants}

Of the five colonized participants, two had detectable colonization at all three postinoculation time points, whereas the remainder were only culture positive at one time point each. The mean colonization density by Day 14 in rechallenge participants (as measured by AUC) was 10.0 cfu days/ml (95\% CI, 0-21.94). This was nonsignificantly lower than the mean density in the same five participants during the first 14 days of the primary challenge (31.01 cfu days $/ \mathrm{ml} ; 95 \%$ CI, 11.08-50.95; $P=0.08$ using Wilcoxon's matched-pairs test).

\section{Anticapsular Antibodies in Rechallenge Participants}

Antibody concentrations before and 14 days after rechallenge are shown in Figure 6 and Table E11 together with the corresponding antibody concentrations from the same participants in the primary challenge for comparison. One participant did not have serum taken on Day 14 after rechallenge. Antibody concentrations did not fall significantly during the interval between the end of the primary challenge and the start of the rechallenge. The baseline antibody concentrations before rechallenge were similar between participants who developed colonization after rechallenge and those who did not (GMT, $3.72 \mu \mathrm{g} / \mathrm{ml}$; 95\% CI, $1.18-11.76$ and GMT, $1.66 \mu \mathrm{g} / \mathrm{ml} ; 95 \% \mathrm{CI}$, $1.17-2.36$, respectively; $P=0.07$ using the Mann-Whitney $U$ test). Antibody concentrations fell in participants who remained uncolonized after rechallenge (GMT at Day 14, $1.53 \mu \mathrm{g} / \mathrm{ml}$; 95\% CI, $1.08-2.17 ; P=0.041$ using Wilcoxon's signed rank test to compare with prechallenge concentrations).

\section{Safety of EHPC in Older Adults}

There were no serious adverse events and no cases of pneumococcal disease. During active surveillance (the week after inoculation), no participants recorded oral temperatures $\geqslant 38^{\circ} \mathrm{C}$. During the primary challenge, seven participants developed symptoms that, when reviewed clinically, were deemed unrelated to study procedures by the investigators; details are summarized in the online supplement. During the rechallenge phase, one colonized participant developed malaise and unilateral otalgia at Day 11 but sought no medical help. The participant attended routinely at Day 14, at which time clinical examination and otoscopy were unremarkable. For precaution, a 5-day course of amoxicillin was prescribed to clear the colonization.

\section{Discussion}

This is the first human bacterial challenge model in older adults. Pneumococcal colonization was safely established at similar rates, densities, and durations as in younger adults $(15,30)$. Colonization rates in the oldest participants in this cohort (ages $70-80 \mathrm{yr}$ ) were nonsignificantly lower than those seen in participants under 50 years 
A

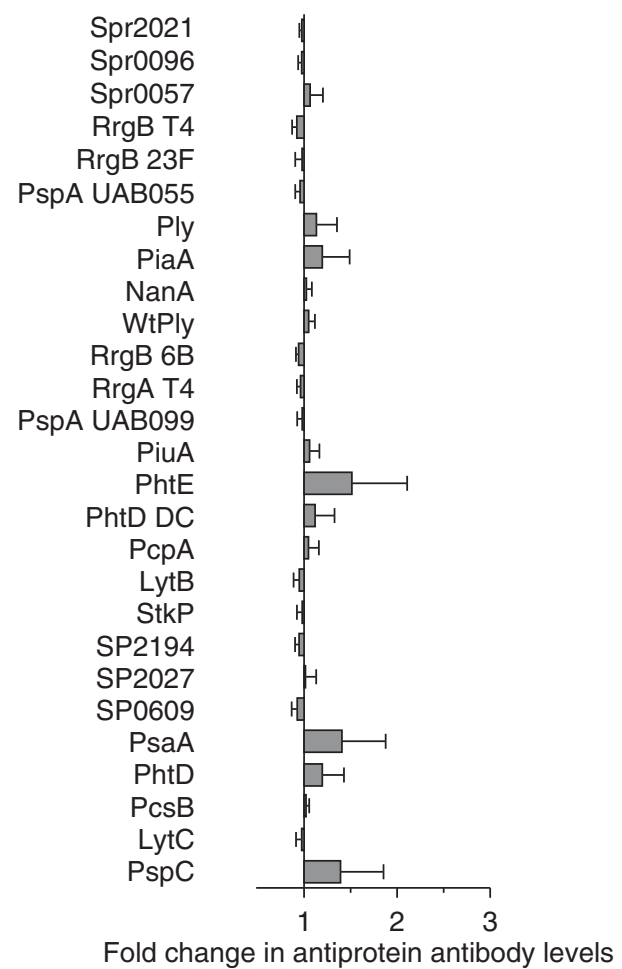

B

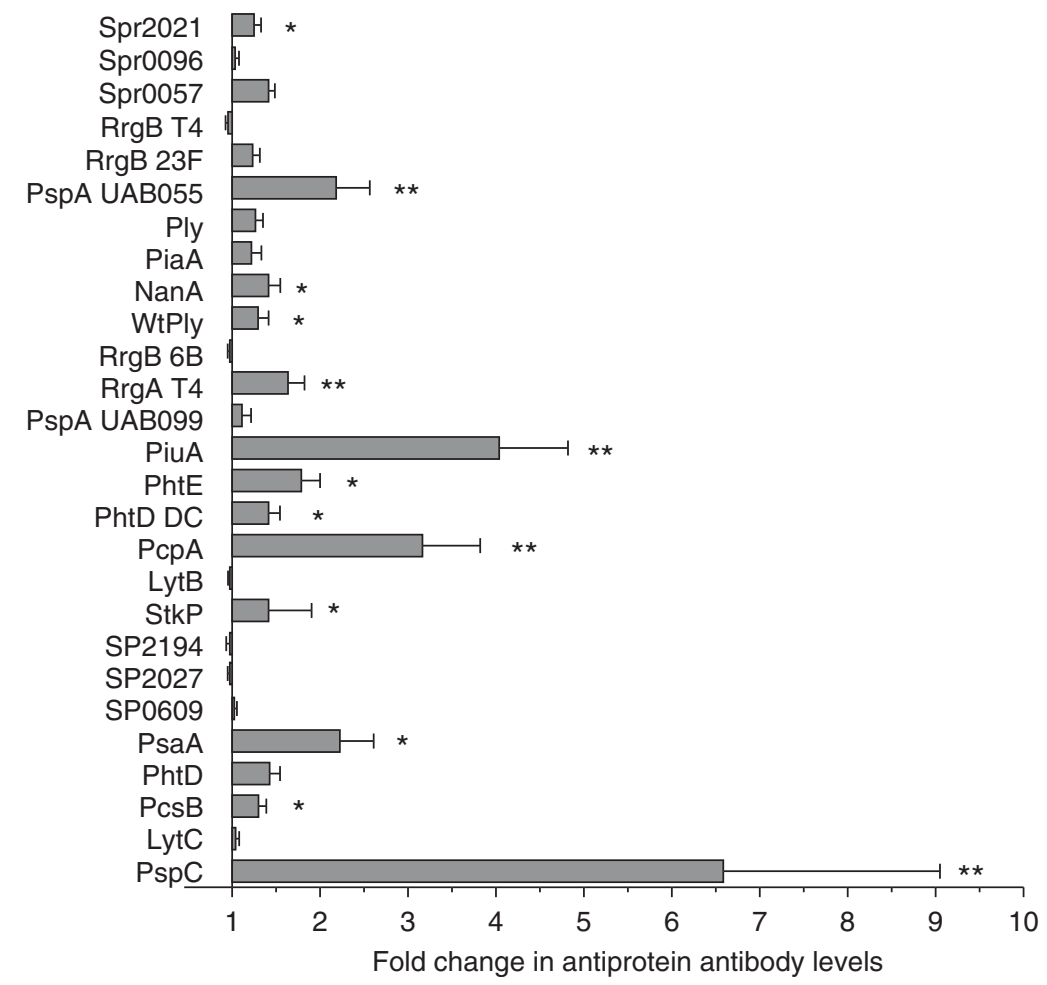

Figure 5. Changes in antibody concentrations against 27 different pneumococcal proteins after nasopharyngeal pneumococcal challenge, measured by multiplex electrochemiluminescence (Meso Scale Discovery), in (A) noncolonized and (B) colonized participants. Antibodies are expressed as fold change between prechallenge baseline and 29 days after inoculation with Streptococcus pneumoniae. Error bars represent mean (SEM). Significant differences in fold change between carriers and noncarriers were analyzed using multivariate regression: ${ }^{*} P<0.05$ and ${ }^{* *} P<0.001$.

old, but low numbers $(n=14)$ preclude definitive conclusions. The ranges and fluctuations in density and variable durations of colonization were similar to

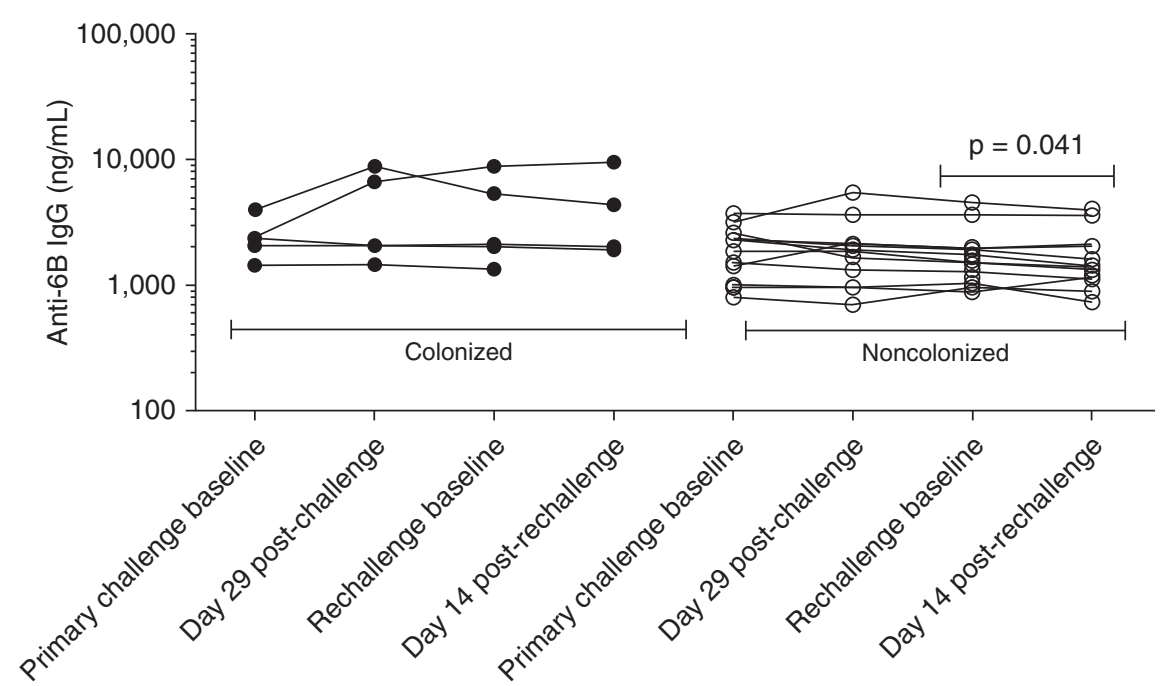

Figure 6. Serum anti-6B capsular antibodies before and after the primary and rechallenge phases of the study. Only participants who were colonized after the primary challenge and returned for rechallenge are shown. They are subdivided into those who did and did not become colonized after rechallenge. Each circle represents an individual participant.

those seen in young participants after both experimental and natural colonization $(30,32)$. PPV23 receipt did not protect against colonization, and baseline antibody concentrations were not different in colonized and noncolonized participants; the latter observation is consistent with previous findings in young adults (15).

When designing this study, we reviewed data from studies conducted in older adults that showed colonization rates $<5 \%(6-8)$. We hypothesized that the older population was somehow less susceptible to pneumococcal colonization than younger adults, a hypothesis that we now reject on the basis of our experimental colonization data. After our study was completed, we collaborated on a meta-analysis that combined studies conducted in participants greater than 60 years old with participantlevel data on those greater than 60 years old from colonization studies of the general population (33). This meta-analysis found widely variable point-prevalence rates (0-39\%) but an overall rate of $9 \%(95 \% \mathrm{CI}$, 6-14). A similar meta-analysis in younger adults has yet to be conducted, but one household study in England found pneumococcal colonization in $8 \%$ of adults (14). These findings suggest that older and younger adults become colonized at similar 
rates, whether in natural or experimental settings, and that older adults' susceptibility to pneumococcal disease is not due to a lack of colonization-mediated boosting.

Indeed, we found that the immunological outcomes after pneumococcal challenge in older adults differ considerably from those in young adults, in whom anticapsular antibody concentrations are raised after experimental colonization and remain unchanged in noncolonized participants (15). Among older adults, antibody concentrations were unchanged after colonization and actually fell in noncolonized participants. Our statistical power to detect age-related immunological trends within the cohort itself was limited by small numbers in each age decile. During rechallenge, we observed no protection against reacquisition of homologous serotype colonization in this older cohort in contrast to the universal protection seen in a previous study of participants aged $<50$ years $(0 / 10$ recolonized) (15).

There are a number of potential explanations for the antibody responses we describe in older adults. Serotype-specific memory B-cell concentrations have been shown to fall after revaccination with PPV23 in older adults (34), and one could speculate that memory B cells may undergo similar terminal differentiation in response to live pneumococcus. Our results may reflect those of vaccine studies, which have demonstrated hyporesponsiveness after repeated doses of polysaccharide in the form of PPV23 (35-37). This hypothesis is supported by our finding that antibody concentrations fell more in PPV23-vaccinated participants than in unvaccinated participants after challenge, although numbers were small in these subgroups, and we did not correct for multiple comparisons. The hyporesponsiveness hypothesis is further supported by the fall in anti-CPS antibodies in noncolonized participants after rechallenge. Alternatively, peripheral antibodies were perhaps sequestered in the nose after pneumococcal challenge, leading to a drop in circulating concentrations. We have previously reported mucosal antibody sequestration by inoculated bacteria in PCV13-vaccinated young adults (preventing colonization via agglutination) but did not observe an effect on peripheral antibody concentrations (24). Equally, in the current study, we did not identify any changes in nasal wash anti-6B IgG concentrations at any time point after the challenge in colonized or noncolonized participants.

Our finding that PPV23 does not prevent colonization contrasts with a recent study in U.S. adults aged $\geqslant 65$ years. The authors followed 100 participants with fortnightly sampling for 1 year and identified a high period prevalence of colonization in the cohort as well as significantly lower colonization in participants who had ever received either pneumococcal vaccine (38). However, most pneumococcal detections were by lyt $A$ quantitative PCR rather than culture, and serotype confirmation was unavailable for the vast majority of isolates. Longitudinal surveillance of U.S. children suggests that any reduction in vaccine-type pneumococcal colonization after vaccination is mostly balanced out by serotype replacement (39). It seems unlikely that PPV23 vaccination of older adults disrupts colonization to such a degree.

Using MSD, we identified boosted antibody concentrations against a number of pneumococcal proteins after colonization. These included PspAUAB055, PspC, and PiuA, which were also boosted after colonization in young adult participants in a previous study using the same MSD methodology (15). This suggests that these proteins are visible to the immune system during colonization and are good markers of exposure. Our finding that baseline antiprotein antibody concentrations were not different between colonized and noncolonized participants suggests that serum antiprotein immunity may not be a major component of antipneumococcal immunity in older adults.

\section{Limitations}

Our participants were carefully screened, which may have resulted in a particularly healthy cohort, characterized by nonsmoking and minimal medical comorbidity. Although this limits the generalizability of our findings, we believed it was necessary to maximize the safety of participants. We defined colonization on the basis of culture positive nasal wash samples rather than molecular testing with typically higher sensitivity $(32,40,41)$. Some authors have argued that the oropharyngeal niche has a higher yield for detecting colonization in older adults than our nasal wash method (9). We did not measure functional antibody activity in this study, but IgG concentrations and serum opsonophagocytic activity are reasonably correlated in older adults (19). The antibody response to colonization may be serotype dependent; population studies have shown a decline in antibodies against certain serotypes (e.g., serotype 3 ) with age but not in other serotypes, including 6B (42). By contrast, an observational study in young adults found that $6 \mathrm{~B}$ colonization did not elicit as strong an antibody response as other serotypes (14). Future research should study the immune response of older adults to challenge with non- $6 \mathrm{~B}$ serotypes.

\section{Conclusions}

New strategies are needed to protect older people against pneumococcal disease. The safety, tolerability, and high rates of experimental colonization seen in this study support the use of EHPC for vaccine testing in this key vulnerable population.

Author disclosures are available with the text of this article at www.atsjournals.org.

Acknowledgment: The authors thank all study participants for their time and commitment. They also thank Catherine Molloy, Kelly Convey, John Blakey, Hassan Burhan, Ben Morton, the members of the data safety and monitoring board (Robert Read and Brian Faragher), the Clinical Research Network North West Coast, the governance staff of the Liverpool School of Tropical Medicine and the Royal Liverpool and Broadgreen University Hospitals National Health Service Trust, and the Royal Liverpool University Hospital Clinical Research Unit. 


\section{References}

1. Welte T, Torres A, Nathwani D. Clinical and economic burden of community-acquired pneumonia among adults in Europe. Thorax 2012;67:71-79.

2. Jain S, Self WH, Wunderink RG, Fakhran S, Balk R, Bramley AM, et al.; CDC EPIC Study Team. Community-acquired pneumonia requiring hospitalization among U.S. adults. N Engl J Med 2015;373:415-427.

3. Bijlsma MW, Brouwer MC, Kasanmoentalib ES, Kloek AT, Lucas MJ, Tanck MW, et al. Community-acquired bacterial meningitis in adults in the Netherlands, 2006-14: a prospective cohort study. Lancet Infect Dis 2016;16:339-347.

4. Thigpen MC, Whitney CG, Messonnier NE, Zell ER, Lynfield R, Hadler JL, et al:; Emerging Infections Programs Network. Bacterial meningitis in the United States, 1998-2007. N Engl J Med 2011;364:2016-2025.

5. Bogaert D, De Groot R, Hermans PW. Streptococcus pneumoniae colonisation: the key to pneumococcal disease. Lancet Infect Dis 2004;4:144-154.

6. Almeida ST, Nunes S, Santos Paulo AC, Valadares I, Martins S, Breia F, et al. Low prevalence of pneumococcal carriage and high serotype and genotype diversity among adults over 60 years of age living in Portugal. PLoS One 2014;9:e90974.

7. Flamaing J, Peetermans WE, Vandeven J, Verhaegen J. Pneumococcal colonization in older persons in a nonoutbreak setting. J Am Geriatr Soc 2010;58:396-398.

8. Becker-Dreps S, Kistler CE, Ward K, Killeya-Jones LA, Better OM, Weber DJ, et al. Pneumococcal carriage and vaccine coverage in retirement community residents. J Am Geriatr Soc 2015;63: 2094-2098.

9. Krone CL, Wyllie AL, van Beek J, Rots NY, Oja AE, Chu ML, et al. Carriage of Streptococcus pneumoniae in aged adults with influenzalike-illness. PLoS One 2015;10:e0119875.

10. van Deursen AM, van den Bergh MR, Sanders EA; Carriage Pilot Study Group. Carriage of Streptococcus pneumoniae in asymptomatic, community-dwelling elderly in the Netherlands. Vaccine 2016;34: 4-6.

11. Boyd AR, Orihuela CJ. Dysregulated inflammation as a risk factor for pneumonia in the elderly. Aging Dis 2011;2:487-500.

12. Haq K, McElhaney JE. Ageing and respiratory infections: the airway of ageing. Immunol Lett 2014;162:323-328.

13. Puchta A, Naidoo A, Verschoor CP, Loukov D, Thevaranjan N, Mandur $\mathrm{TS}$, et al. TNF drives monocyte dysfunction with age and results in impaired anti-pneumococcal immunity. PLoS Pathog 2016;12: e1005368.

14. Goldblatt D, Hussain M, Andrews N, Ashton L, Virta C, Melegaro A, et al. Antibody responses to nasopharyngeal carriage of Streptococcus pneumoniae in adults: a longitudinal household study. J Infect Dis 2005;192:387-393.

15. Ferreira DM, Neill DR, Bangert M, Gritzfeld JF, Green N, Wright AK, et al. Controlled human infection and rechallenge with Streptococcus pneumoniae reveals the protective efficacy of carriage in healthy adults. Am J Respir Crit Care Med 2013;187:855-864.

16. Matanock A, Lee G, Gierke R, Kobayashi M, Leidner A, Pilishvili T. Use of 13-valent pneumococcal conjugate vaccine and 23-valent pneumococcal polysaccharide vaccine among adults aged $\geqslant 65$ years: updated recommendations of the Advisory Committee on Immunization Practices. MMWR Morb Mortal Wkly Rep 2019;68: 1069-1075

17. Joint Committee on Vaccination and Immunisation. Interim JCVI statement on adult pneumococcal vaccination in the UK. London, United Kingdom: Joint Committee on Vaccination and Immunisation; 2015 [accessed 2020 Apr 2]. Available from: https://assets. publishing.service.gov.uk/government/uploads/system/uploads/ attachment data/file/477966/JCVI_pnemococcal.pdf.

18. Simell B, Vuorela A, Ekström N, Palmu A, Reunanen A, Meri S, et al. Aging reduces the functionality of anti-pneumococcal antibodies and the killing of Streptococcus pneumoniae by neutrophil phagocytosis. Vaccine 2011;29:1929-1934.

19. Park S, Nahm MH. Older adults have a low capacity to opsonize pneumococci due to low IgM antibody response to pneumococcal vaccinations. Infect Immun 2011;79:314-320.
20. Bonten MJ, Huijts SM, Bolkenbaas M, Webber C, Patterson S, Gault S, et al. Polysaccharide conjugate vaccine against pneumococcal pneumonia in adults. N Engl J Med 2015;372:1114-1125.

21. Suzuki M, Dhoubhadel BG, Ishifuji T, Yasunami M, Yaegashi M, Asoh N, et al.; Adult Pneumonia Study Group-Japan (APSG-J). Serotypespecific effectiveness of 23-valent pneumococcal polysaccharide vaccine against pneumococcal pneumonia in adults aged 65 years or older: a multicentre, prospective, test-negative design study. Lancet Infect Dis 2017;17:313-321.

22. Ladhani SN, Collins S, Djennad A, Sheppard CL, Borrow R, Fry NK, et al. Rapid increase in non-vaccine serotypes causing invasive pneumococcal disease in England and Wales, 2000-17: a prospective national observational cohort study. Lancet Infect Dis 2018;18:441-451.

23. van Deursen AMM, van Houten MA, Webber C, Patton M, Scott D, Patterson S, et al. The impact of the 13-valent pneumococcal conjugate vaccine on pneumococcal carriage in the Community Acquired Pneumonia Immunization Trial in Adults (CAPiTA) Study Clin Infect Dis 2018;67:42-49.

24. Mitsi E, Roche AM, Reiné J, Zangari T, Owugha JT, Pennington SH, et al. Agglutination by anti-capsular polysaccharide antibody is associated with protection against experimental human pneumococcal carriage. Mucosal Immunol 2017;10:385-394.

25. Moberley S, Holden J, Tatham DP, Andrews RM. Vaccines for preventing pneumococcal infection in adults. Cochrane Database Syst Rev 2013;(1):CD000422.

26. Roestenberg M, Hoogerwerf MA, Ferreira DM, Mordmüller B, Yazdanbakhsh M. Experimental infection of human volunteers. Lancet Infect Dis 2018;18:e312-e322.

27. Mallia P, Footitt J, Sotero R, Jepson A, Contoli M, Trujillo-Torralbo M-B, et al. Rhinovirus infection induces degradation of antimicrobial peptides and secondary bacterial infection in chronic obstructive pulmonary disease. Am J Respir Crit Care Med 2012;186: 1117-1124.

28. Gritzfeld JF, Wright AD, Collins AM, Pennington SH, Wright AK, Kadioglu A, et al. Experimental human pneumococcal carriage. J Vis Exp 2013;(72):50115.

29. Browall S, Norman M, Tångrot J, Galanis I, Sjöström K, Dagerhamn J, et al. Intraclonal variations among Streptococcus pneumoniae isolates influence the likelihood of invasive disease in children. $J$ Infect Dis 2014;209:377-388.

30. Gritzfeld JF, Cremers AJH, Ferwerda G, Ferreira DM, Kadioglu A, Hermans PWM, et al. Density and duration of experimental human pneumococcal carriage. Clin Microbiol Infect 2014;20:01145O1151.

31. Adler H, Nikolaou E, Gould K, Hinds J, Collins AM, Connor V, et al. Pneumococcal colonization in healthy adult research participants in the conjugate vaccine era, United Kingdom, 2010-2017. J Infect Dis 2019;219:1989-1993.

32. Rylance J, de Steenhuijsen Piters WAA, Mina MJ, Bogaert D, French N, Ferreira DM; EHPC-LAIV Study Group. Two randomized trials of the effect of live attenuated influenza vaccine on pneumococcal colonization. Am J Respir Crit Care Med 2019;199:1160-1163.

33. Smith EL, Wheeler I, Adler H, Ferreira DM, Sá-Leão R, Abdullahi O, et al. Upper airways colonisation of Streptococcus pneumoniae in adults aged 60 years and older: a systematic review of prevalence and individual participant data meta-analysis of risk factors. $J$ Infect [online ahead of print] 17 Jun 2020; DOI: 10.1016/j.jinf.2020.06.028.

34. Clutterbuck EA, Lazarus R, Yu LM, Bowman J, Bateman EA, Diggle L, et al. Pneumococcal conjugate and plain polysaccharide vaccines have divergent effects on antigen-specific B cells. J Infect Dis 2012 205:1408-1416.

35. Jackson LA, Neuzil KM, Nahm MH, Whitney CG, Yu O, Nelson JC, et al. Immunogenicity of varying dosages of 7-valent pneumococcal polysaccharide-protein conjugate vaccine in seniors previously vaccinated with 23-valent pneumococcal polysaccharide vaccine. Vaccine 2007;25:4029-4037.

36. Lazarus R, Clutterbuck E, Yu LM, Bowman J, Bateman EA, Diggle L, et al. A randomized study comparing combined pneumococcal conjugate and polysaccharide vaccination schedules in adults. Clin Infect Dis 2011:52:736-742. 
37. O'Brien KL, Hochman M, Goldblatt D. Combined schedules of pneumococcal conjugate and polysaccharide vaccines: is hyporesponsiveness an issue? Lancet Infect Dis 2007;7: 597-606.

38. Branche AR, Yang H, Java J, Holden-Wiltse J, Topham DJ, Peasley $\mathrm{M}$, et al. Effect of prior vaccination on carriage rates of Streptococcus pneumoniae in older adults: a longitudinal surveillance study. Vaccine 2018;36: 4304-4310.

39. Lee GM, Kleinman K, Pelton S, Lipsitch M, Huang SS, Lakoma M, et al. Immunization, antibiotic use, and pneumococcal colonization over a 15-year period. Pediatrics 2017;140:e20170001.
40. Gritzfeld JF, Roberts P, Roche L, El Batrawy S, Gordon SB. Comparison between nasopharyngeal swab and nasal wash, using culture and PCR, in the detection of potential respiratory pathogens. BMC Res Notes 2011;4:122.

41. Sutcliffe CG, Grant LR, Cloessner E, Klugman KP, Vidal JE, Reid R, et al. Association of laboratory methods, colonization density, and age with detection of Streptococcus pneumoniae in the nasopharynx. Am J Epidemiol 2019;188:2110-2119.

42. Simell B, Lahdenkari M, Reunanen A, Käyhty H, Väkeväinen M. Effects of ageing and gender on naturally acquired antibodies to pneumococcal capsular polysaccharides and virulence-associated proteins. Clin Vaccine Immunol 2008;15:1391-1397. 